\title{
Sneddon Syndrome: A Case Report Exploring the Current Challenges Faced with Diagnosis and Management
}

\author{
Jonathan Cleaver ${ }^{\mathrm{a}} \quad$ Mario Teo ${ }^{\mathrm{b}} \quad$ Shelley Renowden ${ }^{\mathrm{c}} \quad$ Keith Miller $^{\mathrm{d}}$ \\ Harsha Gunawardena ${ }^{\mathrm{e}}$ Philip Clatworthy ${ }^{\mathrm{a}}$ \\ aDepartment of Neurology, Southmead Hospital, Bristol, UK; ${ }^{b}$ Department of \\ Neurosurgery, Southmead Hospital, Bristol, UK; ' ${ }^{c}$ Department of Neuroradiology, \\ Southmead Hospital, Bristol, UK; dDepartment of Pathology, Southmead Hospital, \\ Bristol, UK; eDepartment of Rheumatology, Southmead Hospital, Bristol, UK
}

\section{Keywords}

Antiphospholipid syndrome - Livedo reticularis - Livedo racemosa · Sneddon syndrome ·

Stroke $\cdot$ Vasculopathy

\begin{abstract}
Sneddon syndrome (SS) is a rare medium-vessel vasculopathy which characteristically presents with livedo racemosa (LR) and complications such as strokes. This case report describes a female presenting acutely with a stroke and, initially, no evidence of LR. Her antiphospholipid antibodies were negative, and her neuroimaging revealed multiple territory strokes with extensive vasculopathy and fragile neo-formed vessel collateralisation. She had progressive memory loss and multiple transient ischaemic attacks on a background of established infarctions. SS should be considered in any idiopathic medium-vessel vasculopathy despite the absence of LR. Medical therapy can be challenging and the importance of antiphospholipid status in risk stratifying anticoagulation against antiplatelet therapy is discussed with a proposed
\end{abstract}




\section{Case Reports in Neurology}

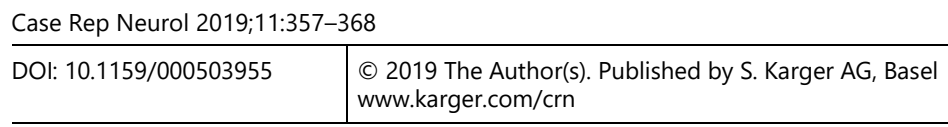

Cleaver et al.: Sneddon Syndrome: Exploring Diagnosis and Management

rheumatology management strategy. The medical option of hydroxychloroquine should be considered in all patients in view of its anti-thrombotic properties and efficacy in diseases such as systemic lupus erythematosus and antiphospholipid syndrome with the suggestion that SS may be a forme fruste of these diseases. Neurosurgical options should be considered for recurrent transient neurological symptoms. For our patient, this included an extracranial to intracranial bypass via a radial artery graft for haemodynamic stroke management confirmed on SPECT imaging. The traditional hallmark of SS has previously been LR. This case highlights an atypical presentation stressing the importance of diagnostic vigilance in a patient with an idiopathic medium-vessel vasculopathy, together with balancing the medical risk of antiplatelet therapy, anticoagulation and thrombolysis whilst revealing possible neurosurgical options in select SS patients.

(C) 2019 The Author(s)

Published by S. Karger AG, Basel

\section{Introduction}

Sneddon syndrome (SS) is a rare non-inflammatory arteriopathy which is characterised by cerebrovascular disease and concomitant livedo racemosa (LR) [1]. It has an incidence of 4 in 1 million, generally affects females (34 of 46 patients in one study [2] or 5 of 6 patients in Sneddon's original report [3]) between 20 and 42 years of age and rarely has a presumed autosomal dominant familial predisposition [4].

SS can be classified into three forms: these include idiopathic SS without antiphospholipid (aPL) antibodies, primary aPL syndrome-related SS and systemic lupus erythematosus-related SS in the presence or absence of aPL antibodies [2].

Its pathophysiology is poorly understood with the main theories postulating a primarily thrombotic focus [5], a cardio-embolic aetiology caused by associated valvular disease [6] or inherited/acquired endothelial dysfunction [7].

The hallmark of the disease is typically LR, a persistent and pathological form of livedo reticularis, which is caused by intimal thickening of the small deep dermal arteries. This leads to reduced blood flow and thus oxygen tension at the skin peripheries [6]. LR provides a valuable diagnostic clue in the work-up of SS; however, the rare onset of neurological symptoms prior to the development of LR is possible [7].

\section{Case Study}

A 49-year-old right-handed retired Caucasian female was admitted to the stroke ward following a 24-h history of expressive dysphasia, confusion, and a right frontal headache of gradually increasing moderate severity. On closer questioning, she reported new intermittent generalised weakness and paraesthesia affecting her hands and feet.

Past medical history included hypertension, hypercholesterolaemia, migraine and psoriasis. Despite previous strokes with some residual left-sided weakness, she continued to smoke (30 pack-years) and her only medication was ramipril. Prior to her stroke, she lived independently with her daughter. She also had a strong family history of stroke, including her 
cousin (onset at the age of 30-years), paternal uncle and grandmother (the latter 2 at unknown ages).

On neurological examination, apart from the pre-existing mild left-sided weakness ( $4 / 5$ power on the Medical Research Council scale in the upper and lower limbs), she was also newly dysphasic and exhibited right-sided sensory inattention. She was normotensive and cardiopulmonary examination was normal. Initially, there was no LR evident on examination of the skin and the patient denied the presence of a rash on direct questioning. She was alert, but with severe cognitive impairment (Montreal Cognitive Assessment [MoCA] score 3/30).

Non-contrast CT imaging revealed multi-focal territory established infarcts (Fig. 1a) with an acute diffusion-weighted imaging positive left posterior temporal lobe infarct in the territory of the posterior cerebral artery confirmed on MRI (Fig. 1b). CT angiography revealed multiple stenotic lesions of the bilateral carotid, right distal middle cerebral, left M2 middle cerebral and left posterior cerebral arteries (Fig. 1c, d). Further visualisation of the vessels through digital subtraction angiography confirmed multiple cerebral artery stenoses with new vessel collateralisation.

Due to the multi-territory stenoses and time from symptom onset to presentation in hospital, neither thrombolysis nor thrombectomy were feasible and a working diagnosis of medium-vessel vasculopathy of uncertain aetiology was made. Conservative medical treatment was initiated with dual antiplatelet therapy (aspirin and clopidogrel) for 1 month followed by lifelong clopidogrel monotherapy. High-dose atorvastatin was administered and smoking cessation strongly advised.

\section{Differential Diagnoses}

Differential diagnoses of a medium-vessel vasculopathy included SS, medium-vessel vasculitis, atherosclerosis, fibromuscular dysplasia (FMD) and Behçet's disease. Atherosclerosis was a possibility but weakened by the presence of gross intracranial circulatory abnormalities more insinuative of an obliterative arteriopathy. Vasculitis was possible but unlikely in view of the lack of preceding prodromal symptoms, renal involvement with a normal urine dip and no aneurysms found on abdominal CT imaging. Intracranial angiography was discussed with the multi-disciplinary team and there were no typical features of FMD such as a "string of beads" appearance and no intra-abdominal stenoses. There was no history of mouth or genital ulcers, iritis, or prior venous thromboembolisms to suggest Behçet's disease.

Deficiency of adenosine deaminase 2 (DADA2) due to mutations of the CECR1 (cat eye syndrome chromosome region, candidate 1) gene has been linked with vasculopathies, LR and early-onset lacunar strokes [8]. DADA2 typically causes strokes in the first decade associated with hepatosplenomegaly or hypogammaglobulinaemia, making it unlikely.

\section{Additional Investigations}

Further tests were ordered in an attempt to elucidate the cause. Baseline bloods revealed a normal full blood count, electrolytes and liver function. A vasculitic screen including antinuclear antibodies, double-stranded DNA, aPL antibodies (lupus anticoagulant, anti-cardiolipin and beta-2 glycoprotein-1 antibody), extractable nuclear antigen, anti-neutrophil cytoplasmic antibody, rheumatoid factor and complement levels were negative or normal. A myeloma screen including serum and urine protein electrophoresis, serum-free light chains and Bence Jones protein was negative. Hepatitis $\mathrm{C}$ antibody was positive but polymerase chain 


\section{Case Reports in Neurology}

reaction was negative indicating past infection now cleared. Cerebrospinal fluid examination was unremarkable. An electrocardiogram (ECG) revealed normal sinus rhythm which was later corroborated on a 7-day ambulatory ECG. A transthoracic echocardiogram revealed no vegetations or thrombi and there were no valvular abnormalities.

\section{Diagnosis}

The opinion of a dermatologist was sought for skin examination and consideration of a biopsy if evidence of a skin abnormality suggestive of LR was found. Idiopathic SS was suspected in view of the angiographic appearances, clinical presentation with multi-territory infarcts and lack of an alternative identified cause. Causes of LR and differentials of skin lesions and strokes are summarised in Table 1 and Table 2, respectively.

She was seen some months later and found to have subtle changes on her upper thighs bilaterally consistent with LR (Fig. 2a). Three deep dermal biopsies taken, for optimal sensitivity testing, demonstrated marked intimal dermal artery proliferation and luminal narrowing with no active vasculitis confirming the diagnosis of idiopathic SS (Fig. 2b).

\section{Additional Treatments to Consider}

Potential treatment was discussed with rheumatology, and hydroxychloroquine (HCL) commenced due to its effects on endothelial function, its antithrombotic properties and efficacy in the treatment of systemic lupus erythematosus and antiphospholipid syndrome (APS). High-dose anticoagulation was avoided in our patient due to a high bleeding risk associated with a fragile neo-formed anterior collateral circulation supply together with the negative aPL antibodies.

\section{Follow-Up and Post-Operative Outcome}

Over the subsequent months, she continued to complain of intermittent hands/feet numbness, expressive dysphasia, headache and worsening memory impairment - although her MoCA score had improved to 10/30 and stabilised - despite optimal medical therapy. An interval MRI of the brain, 8 months following her admission, revealed progressive cerebral ischaemic changes.

She was reviewed by neuropsychology, 13 months later, for formal cognitive assessment following medical therapy, which showed significant global cognitive impairment across multiple domains including visuospatial, language, attention, digit span, trail-making test, verbal fluency, processing speed, immediate and delayed memory. As a result, she required support from her daughter in the form of regular supervision, shopping, cooking and cleaning.

The combination of steno-occlusive disease with transient neurological symptoms and cognitive impairment raised the suspicion of haemodynamic ischaemic events, triggering referral to neurosurgery for further investigation. A SPECT (single positron emission CT) study was then performed which revealed impaired reserve in the frontal branch of the left middle cerebral artery in keeping with haemodynamic ischaemic events anatomically consistent with progressive memory impairment over the course of the year. One month later, she underwent an elective extracranial to intracranial (EC-IC) bypass using a radial artery graft to augment her left hemispheric cerebral blood flow to improve her cognition (Fig. 3a-d).

Post-operatively, she reported subjective functional improvement with a good recovery. She was able to walk her dog, an activity she enjoyed but struggled with pre-operatively. Her 
repeated neurological events secondary to impaired cerebral haemodynamic also stopped postoperatively.

However, 3 months after her EC-IC bypass the patient was re-admitted following a contralateral acute stroke causing left-sided hemiparesis. This occurred 18 months after her initial diagnosis, which demonstrated the severity of her widespread vasculopathy. In light of this, she is awaiting further discussion regarding the appropriateness of a bypass operation on the contralateral side in view of her limited medical options.

\section{Discussion}

\section{Diagnostic Dilemma}

LR is historically the hallmark of SS, described by the British Dermatologist Ian Sneddon in 1965 [3]. It is described as an erythematous-violaceous rash which consists of a network of broken irregular circles, persistent on rewarming. It is distinguished from livedo reticularis which has a continuous net-like pattern and a tendency to reverse on rewarming. In a systematic review of case series, LR was present on the limbs in all patients, followed by the trunk (87\%), buttocks (71\%), hands/feet (56\%) and face (16\%) [2].

The differential diagnoses of a medium-vessel (major intracerebral vessel) vasculopathy are limited including atherosclerosis, medium-vessel vasculitis, FMD, Behçet's disease, DADA2 and SS. Therefore, SS should be considered as a potential diagnosis in all cases without a determined cause; even in the absence of LR and aPL antibodies which are positive variably in the literature from 0 to $85 \%[10,11]$. Follow-up dermatological examinations should be undertaken as LR may appear later.

LR might not be the initial presentation in patients with SS as highlighted, but with close surveillance we were able to establish the diagnosis with delayed emergence of such lesion. Alternatively, the initial examination may have missed the underlying LR. It can be subtle and on clothed-covered areas which may not be fully exposed routinely. We suggest exposing these areas and asking the patient for any notable skin changes; although the reliability of this history in the context of cognitive impairment may be limited. Our patient exhibited key features of SS additional to LR including multiple strokes and intracranial stenoses classically involving the perfusion zones of the middle and posterior cerebral arteries $[1,10]$ together with marked cognitive dysfunction in a young patient, heightening suspicion. Cognitive dysfunction has been shown to occur in $77 \%$ of SS and can ultimately lead to early-onset dementia stressing the need for targeted and optimal management $[1,11]$.

The management for these patients remains challenging with episodes of recurrent strokes due to steno-occlusive intracranial circulations. Medical management is limited in patients without aPL antibodies; thus, we highlight the supplementary possibility of surgical options in select patients and the stepwise approach to achieving this.

\section{Medical Management}

The choice of anti-thrombotic treatment was carefully considered. The evidence for the therapeutic benefit of high-dose anticoagulation (target INR >3) over antiplatelet therapy for aPL antibody-negative SS is weak. A prospective study involving 46 patients followed over 6 years found no significant difference between the number of cerebrovascular events per year 


\section{Case Reports in Neurology}

Case Rep Neurol 2019;11:357-368

DOI: $10.1159 / 000503955$

(c) 2019 The Author(s). Published by S. Karger AG, Basel www.karger.com/crn

Cleaver et al.: Sneddon Syndrome: Exploring Diagnosis and Management

for antiplatelet therapy (0.08) and that for high-dose anticoagulation with warfarin $(0.06)$ in aPL-negative individuals [2].

In the absence of aPL antibodies, we found no clear evidence in favour of full-dose anticoagulation over antiplatelet therapy, but the choice of anti-thrombotic treatment must be considered on an individual basis. In our case, the decision to avoid full-dose anticoagulation was strengthened by the presence of fragile newly formed vessels creating a secondary moyamoya phenomenon. This is presumed secondary to widespread vasculopathy and resultant chronic hypoxaemia which may have a higher probability of bleeding [7]. Treatment of SS should, therefore, be tailored to the individual due to small patient numbers and significant variation meaning there is limited high-level evidence in the literature. We recommend repeating the aPL antibody tests if the clinical course changes or the patient develops a new vascular event because positive antibodies will alter the risk of ischaemic stroke and may affect the choice of anti-thrombotic treatment.

Thrombolysis in SS has not been well studied. In one case report a patient received recombinant tissue plasminogen activator with significant neurological improvement and no bleeding complications [12]. The report suggests performing perfusion imaging and MRI including susceptibility-weighted imaging sequences to identify acute ischaemic lesions and penumbra whilst excluding microbleeds. It suggests favourable outcomes with intravenous thrombolysis may occur if the mean transit time is reduced with normal cerebral blood volume in the area correlating with the acute diffusion-weighted imaging region, suggesting "an already perfused' ischaemic lesion. However, this report demonstrated no evidence of an SSassociated moyamoya phenomenon or microbleeds which likely increases the risk of intracerebral haemorrhage [5, 7]. Overall, thrombolysis needs to be balanced against the likelihood of disability from the acute ischaemic event, with consideration of MRI and perfusion imaging if available acutely, whilst involving patients and relatives in this treatment option.

The rationale for HCL in our patient was judged on eminence-based practice through its efficacy in treating systemic lupus erythematosus and APS. These are all forms of arteriopathy, and it has been postulated that SS is a forme fruste of APS (or a seronegative APS) for which we have not identified the antigen targets and thus the specific autoantibody [11]. Furthermore, HCL has anti-thrombotic properties through inhibiting prothrombotic tissue factor and tumour necrosis factor alpha [13]. It concomitantly prevents endothelial dysfunction by improving endothelium-dependent dilatation via nitric oxide synthase coupling and oxidative stress reduction in patients with aPL antibodies [14].

Our patient explored 3 months of optimal medical therapy before being referred for a SPECT scan. It was by this point that multi-disciplinary team discussions advised for the possibility of neurosurgical interventions if there were to be haemodynamic ischaemic events.

\section{Surgical Management}

In view of the multiple strokes, transient recurrent neurological deficits and findings from the SPECT study in keeping with haemodynamic ischaemic events, she underwent an EC-IC bypass using a radial artery graft to augment her left hemispheric cerebral blood flow (Fig. 3). As a result of the proximal vessel occlusions and steno-occlusive disease affecting the medium vessels she was not suitable for an endovascular stent insertion; leaving EC-IC bypass surgery her only viable surgical option. 
Despite the risks of this invasive procedure, a recent meta-analysis, showed that surgical management using an EC-IC arterial bypass in patients with moyamoya disease significantly lowered the incidence of primary end points (including all-cause mortality, completed strokes and re-bleeds) compared with non-surgical therapy (OR 0.35, 95\% CI 0.15-0.84, I $\mathrm{I}^{2}=0 \%$ ) [15]. In this case of SS with moyamoya type intracranial steno-occlusive vasculopathy, EC-IC bypass should therefore be considered, as a multi-disciplinary team, where clinico-radiological haemodynamic ischaemic events predominate in patients deemed suitable for this operation, despite best medical therapy.

We proceeded with a unilateral bypass in view of the SPECT study findings of a poorer cerebrovascular reserve on the left hemisphere, despite angiography showing bilateral intracranial vessel steno-occlusions affecting multiple territories with evidence of bi-hemispheric infarcts. Unfortunately, 3 months after an uneventful left-sided EC-IC bypass, despite making good clinical progress, she developed a contralateral stroke. We are in the process of re-evaluating her for surgical consideration.

\section{Conclusions}

In summary, this case report highlights the diagnostic dilemmas, current evidence for medical therapy and the neurosurgical interventions possible for SS. We stress the importance of keeping a high diagnostic suspicion of SS for any young patient with multiple territory strokes or severe cognitive changes and a medium-vessel vasculopathy. We also triage the management of SS, exploring the most up-to-date optimal medical treatment together with the novel consideration of neurosurgical interventions if haemodynamic ischaemic events persist.

\section{Statement of Ethics}

The authors confirm obtaining written consent from the patient for publication of the manuscript.

\section{Disclosure Statement}

The authors declare that the research was conducted in the absence of any commercial, or financial relationships that could be construed as a potential conflict of interest.

\section{Funding Sources}

The authors received no funding for the publication of the manuscript. 


\section{Author Contributions}

J.C., P.C., M.T. and H.G. contributed to the manuscript development, rationale and patient management. K.M. and S.R. contributed to the manuscript images and descriptions.

\section{References}

1 Boesch SM, Plörer AL, Auer AJ, Poewe W, Aichner FT, Felber SR, et al. The natural course of Sneddon syndrome: clinical and magnetic resonance imaging findings in a prospective six year observation study. J Neurol Neurosurg Psychiatry. 2003 Apr;74(4):542-4.

2 Francès C, Papo T, Wechsler B, Laporte JL, Biousse V, Piette JC. Sneddon syndrome with or without antiphospholipid antibodies. A comparative study in 46 patients. Medicine (Baltimore). 1999 Jul;78(4): 209-19.

3 Sneddon IB. Cerebrovascular lesions and livedo reticularis. Br J Dermatol. 1965 Apr;77(4):180-5.

4 Llufriu S, Cervera A, Capurro S, Chamorro A. Familial Sneddon's syndrome with microbleeds in MRI. BMJ Case Rep. 2009;2009:bcr2007131912.

5 Asherson RA, Khamashta MA, Ordi-Ros J, Derksen RH, Machin SJ, Barquinero J, et al. The "primary" antiphospholipid syndrome: major clinical and serological features. Medicine (Baltimore). 1989 Nov;68(6):366-74.

6 Fierini F, Barilaro A, Giambene B, Carlucci G, Grandi V, Maio V, et al. Moyamoya in a patient with Sneddon's syndrome. Clin Neurol Neurosurg. 2015 Feb;129:34-6.

7 Marianetti M, Mina C, Marchione P, Giacomini P. Sneddon's Syndrome presenting with topographic disorientation. J Clin Neurosci. 2011 Jul;18(7):980-1.

8 Zhou Q, Yang D, Ombrello AK, Zavialov AV, Toro C, Zavialov AV, et al. Early-onset stroke and vasculopathy associated with mutations in ADA2. N Engl J Med. 2014 Mar;370(10):911-20.

9 Samanta D, Cobb S, Arya K. Sneddon syndrome: A comprehensive overview. J Stroke Cerebrovasc Dis. 2019 Aug;28(8):2098-108.

10 Tourbah A, Piette JC, Iba-Zizen MT, Lyon-Caen O, Godeau P, Francès C. The natural course of cerebral lesions in Sneddon syndrome. Arch Neurol. 1997 Jan;54(1):53-60.

11 Tietjen GE, Al-Qasmi MM, Gunda P, Herial NA. Sneddon's syndrome: another migraine-stroke association? Cephalalgia. 2006 Mar;26(3):225-32.

12 Sun J, Zhang F, Gao F, Wang J, Selim M, Lou M. Intravenous thrombolysis in Sneddon's syndrome. J Clin Neurosci. 2012 Feb;19(2):326-8.

13 Zelger B, Sepp N, Stockhammer G, Dosch E, Hilty E, Ofner D, Aichner F, Fritsch O. Sneddon's syndrome. A long-term follow-up of 25 patients. Arch Dermatol. 1993;129(4):437-47.

14 Müller-Calleja N, Manukyan D, Ruf W, Lackner K. Mechanism of action of hydroxychloroquine in antiphospholipid syndrome. Blood. 2016;128(22):5023.

15 Liu L, Su SW, Sun HY. Safety of Extracranial-Intracranial Arterial Bypass in the Treatment of Moyamoya Disease. J Craniofac Surg. 2017 Sep;28(6):e522-7. 


\section{Case Reports in Neurology}

Case Rep Neurol 2019;11:357-368

DOI: $10.1159 / 000503955$

(C) 2019 The Author(s). Published by S. Karger AG, Basel www.karger.com/crn

Cleaver et al.: Sneddon Syndrome: Exploring Diagnosis and Management
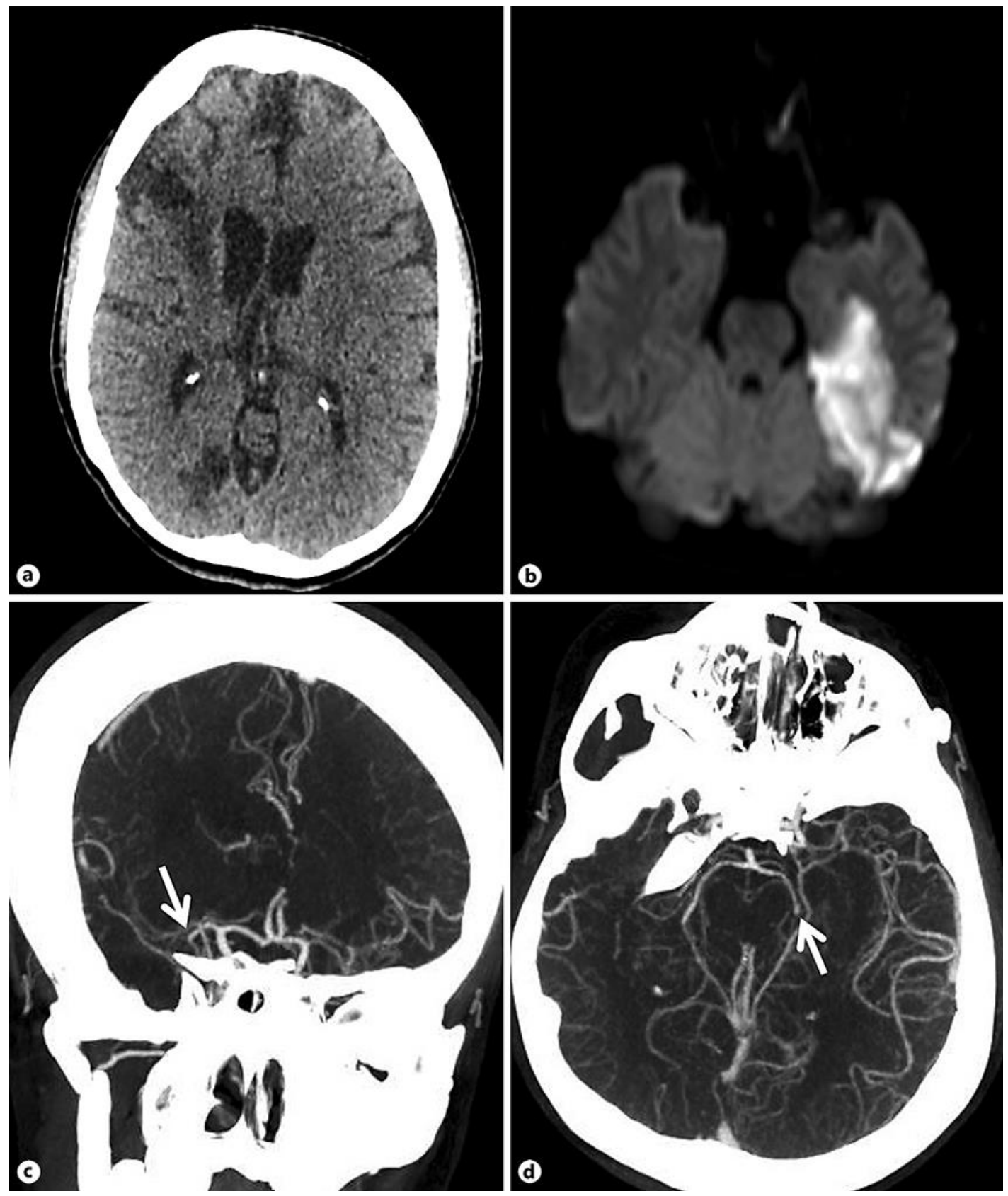

Fig. 1. a Initial neuroimaging: axial non-contrast cranial computerised tomography (CT) scan demonstrates two areas of established infarctions in the right frontal lobe and right medical occipital lobe and mild generalised volume loss. b Cranial magnetic resonance imaging (MRI) scan: diffusion-weighted axial image confirms an area of acute infarction involving the left posterior temporal lobe, in the posterior cerebral arterial territory. Maximum image projection CT angiogram images: oblique coronal (c) and oblique axial (d), show distal right middle cerebral artery occlusion (arrow, c) and left middle cerebral artery M2 branch occlusion. The left posterior cerebral artery is occluded (arrow, d). 


\section{Case Reports in Neurology}
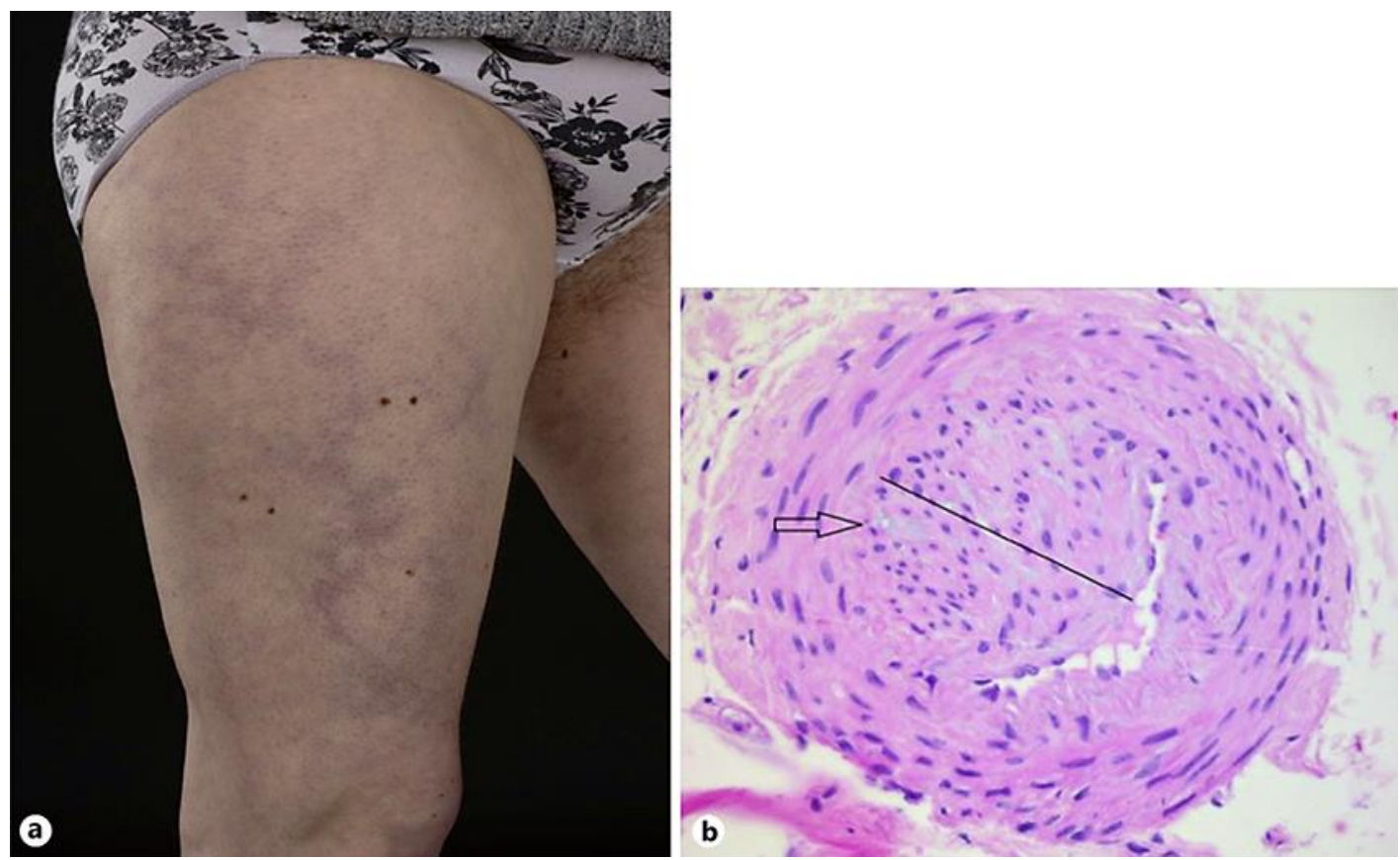

Fig. 2. Macroscopic and microscopic appearances of LR. LR rash (a), present on both upper thighs. Note the broken circular erythematous-violaceous pattern that is persistent on rewarming. b A high-power $(\times 400)$ image of a thickened artery with no inflammation but marked intimal proliferation (line) and narrowing of the lumen characteristic of SS (arrow indicates the elastic lamina). 


\section{Case Reports in Neurology}
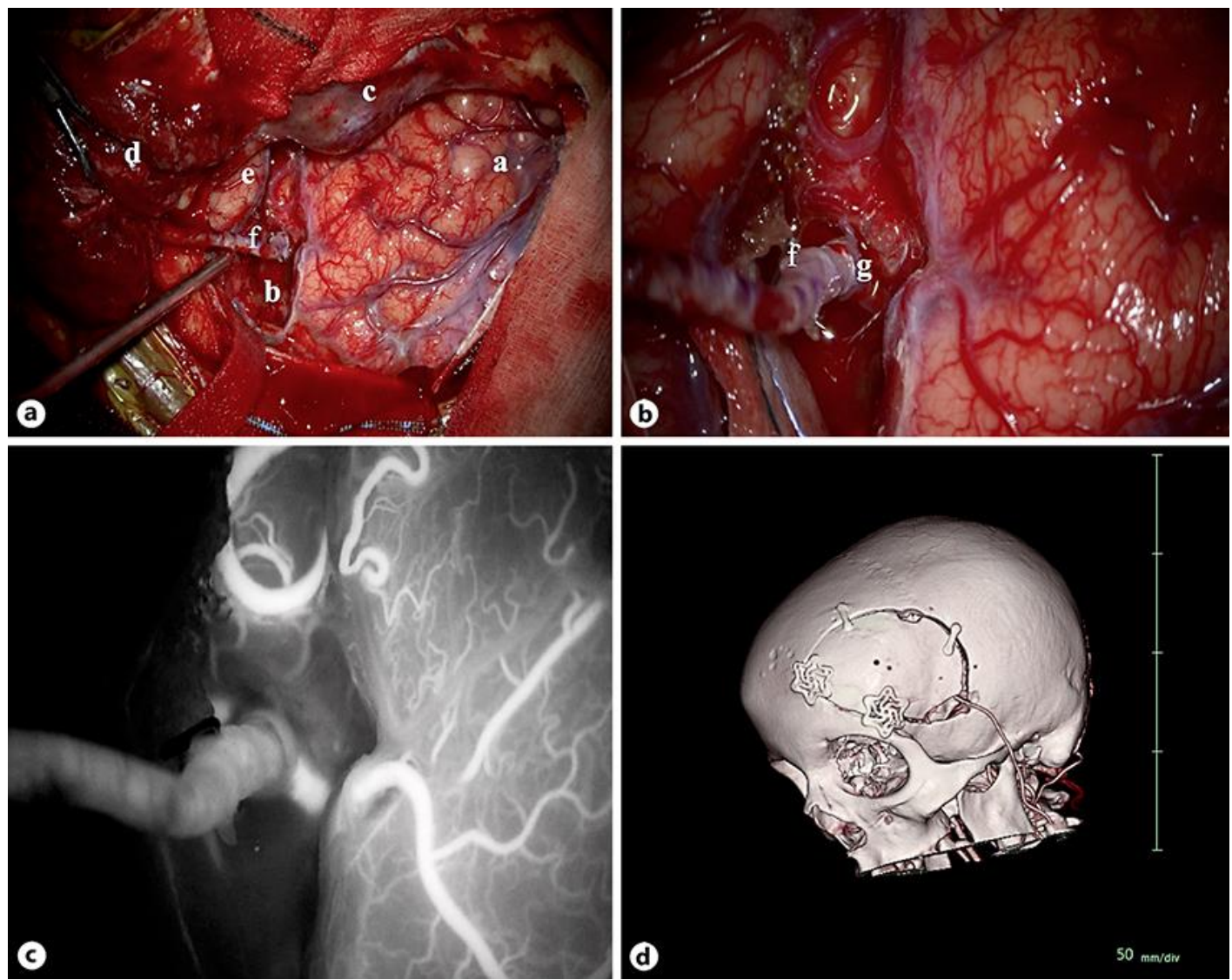

Fig. 3. Intraoperative EC-IC bypass images: zoom out (a) and zoom in (b) intraoperative views of the left hemisphere demonstrating the radial artery bypass to the recipient left M2 middle cerebral artery. Intraoperative indocyanine green angiogram (c) confirming bypass graft patency and robust blood flow supplying the left cerebral hemisphere; especially the left frontal region. 3D reconstruction image (d) demonstrating the external anatomy in relation to the radial artery bypass graft. $a$, frontal lobe; $b$, sylvian fissure; c, sphenoid wing; d, temporalis muscle; e temporal lobe; f radial artery bypass graft; g M2 middle cerebral artery recipient. 
Cleaver et al.: Sneddon Syndrome: Exploring Diagnosis and Management

Table 1. Differential diagnosis for secondary associations with LR

\begin{tabular}{ll} 
Haematological & $\begin{array}{l}\text { Polycythaemia rubra vera, cryoglobulinaemia, multiple myeloma, cold agglutinin } \\
\text { disease, protein C and S deficiency, antithrombin III deficiency, disseminated intra- } \\
\text { vascular coagulation, haemolytic uraemic syndrome, deep venous thrombosis }\end{array}$ \\
\hline Rheumatological & $\begin{array}{l}\text { SS, anti-phospholipid syndrome, systemic lupus erythematosus, dermatomyositis, } \\
\text { rheumatoid arthritis, polyarteritis nodosa, granulomatosis with polyangiitis, giant } \\
\text { cell arteritis, Sjogren syndrome }\end{array}$ \\
\hline Infection & $\begin{array}{l}\text { Mycoplasma, syphilis, tuberculosis, viral infections (parvovirus and hepatitis C), } \\
\text { bacterial infections (meningococcal and streptococcal), rheumatic fever }\end{array}$ \\
\hline Malignancy & Renal cell carcinoma, lymphoma, acute lymphocytic leukaemia, breast cancer \\
\hline Drugs & Minocycline, gemcitabine, non-steroidal anti-inflammatories, catecholamines \\
\hline Miscellaneous & $\begin{array}{l}\text { Cholesterol emboli, septic emboli, thromboangiitis obliterans, pancreatitis, congeni- } \\
\text { tal hypogammaglobulinaemia }\end{array}$ \\
\hline Congenital & Physiological congenital cutis marmorata \\
\hline
\end{tabular}

Adapted from Samanta et al. [9].

Table 2. Differential diagnosis for diseases that can present with rashes which are associated with an increased risk of stroke

\begin{tabular}{ll}
\hline Inherited & Fabry's disease, Divry van Bogaert Syndrome \\
\hline Haematological & $\begin{array}{l}\text { Hyperviscosity syndromes (polycythaemia rubra vera, cryoglobulinaemia, multiple } \\
\text { myeloma, cold agglutinin disease), thrombophilias (protein C and S deficiency, an- } \\
\text { tithrombin III deficiency) }\end{array}$ \\
\hline Rheumatological & $\begin{array}{l}\text { Vasculitis, systemic lupus erythematosus, anti-phospholipid syndrome, Sjogren } \\
\text { syndrome, Behçet's, rheumatoid arthritis, deficiency of adenosine deaminase } 2 \\
\text { (DADA 2) }\end{array}$ \\
\hline Malignancy & Lymphoma \\
\hline Miscellaneous & Cholesterol emboli, septic emboli, thromboangiitis obliterans, pancreatitis \\
\hline
\end{tabular}

